\title{
Inability of HDL from type 2 diabetic patients to counteract the inhibitory effect of oxidised LDL on endothelium-dependent vasorelaxation
}

Received: 3 August 2005 / Accepted: 23 February 2006 / Published online: 5 April 2006

C) Springer-Verlag 2006

\begin{abstract}
Aims/hypothesis: In healthy normolipidaemic and normoglycaemic control subjects, HDL are able to reverse the inhibition of vasodilation that is induced by oxidised LDL. In type 2 diabetic patients, HDL are glycated and more triglyceride-rich than in control subjects. These alterations are likely to modify the capacity of HDL to reverse the inhibition of vasodilation induced by oxidised LDL. Subjects and methods: Using rabbit aorta rings, we compared the ability of HDL from 16 type 2 diabetic patients and 13 control subjects to suppress the inhibition of vasodilation that is induced by oxidised LDL. Results: Oxidised LDL inhibited endothelium-dependent vasodilation (maximal relaxation $[$ Emax $]=58.2 \pm$ 14.6 vs $99.3 \pm 5.2 \%$ for incubation without any lipoprotein, $p<0.0001$ ). HDL from control subjects significantly reduced the inhibitory effect of oxidised LDL on vasodilatation (Emax $=77.6 \pm 12.9$ vs $59.5 \pm 7.7 \%, p<0.001)$, whereas HDL from type 2 diabetic patients had no effect $($ Emax $=52.4 \pm 20.4$ vs $57.2 \pm 18.7 \%$, NS). HDL triglyceride content was significantly higher in type 2 diabetic patients than in control subjects $(5.3 \pm 2.2$ vs $3.1 \pm 1.4 \%, p<0.01)$ and was highly inversely correlated to Emax for oxidised LDL+HDL in type 2 diabetic patients $(r=-0.71, p<0.005)$. Conclusions/interpretation: In type 2 diabetes mellitus, the ability of HDL to counteract the inhibition of endothelium-dependent vasorelaxation induced by oxidised
\end{abstract}

\footnotetext{
L. Perségol · B. Vergès $\cdot$ M. Foissac .

P. Gambert · L. Duvillard $(\bowtie)$

INSERM U498, Hôpital du Bocage,

BP 77908, 21079 Dijon Cédex, France

e-mail: laurence.duvillard@chu-dijon.fr

Tel.: +33-380293646

Fax: $+33-380293661$

L. Perségol · B. Vergès · M. Foissac ·

P. Gambert · L. Duvillard

University of Burgundy,

IFR 100, Dijon, France
}

B. Vergès

Department of Endocrinology and Metabolic Diseases, CHU, Bocage Hospital,

Dijon, France
LDL is impaired and is inversely correlated with HDL triglyceride content. These findings suggest that HDL are less atheroprotective in type 2 diabetic patients than in control subjects.

Keywords HDL · Oxidised LDL · Triglyceride · Type 2 diabetes mellitus - Vasodilatation

Abbreviations apo: apolipoprotein $\cdot d$ : density $\cdot$ eNOS: endothelial NO synthase - NO: nitric oxide - ox-LDL: oxidised LDL - PAF-AH: platelet-activated factor-acetyl hydrolase - PON: paraoxonase - TBS: Tris buffer saline . TG: triglyceride

\section{Introduction}

Type 2 diabetic patients present a significantly higher risk for atherosclerosis than nondiabetic subjects. The quantitative and qualitative lipoprotein abnormalities frequently observed in these patients are likely to play a central role in the premature development of atherosclerotic lesions. Quantitative abnormalities observed in type 2 diabetic patients are hypertriglyceridaemia and decreased plasma HDL cholesterol concentration. The main qualitative abnormalities consist of the glycation and a triglyceride (TG) enrichment of the different lipoprotein subclasses $[1,2]$.

HDL particles have multiple antiatherosclerotic properties. One of them is their beneficial effect on endotheliumdependent vasorelaxation. The intravenous administration of reconstituted HDL either to hypercholesterolaemic men or to heterozygotes deficient in ATP-binding cassette A1, both of whom had initially impaired endothelium-dependent vasorelaxation, restored normal vasorelaxation $[3,4]$. More particularly, ex vivo experiments on isolated strips of rabbit thoracic aorta showed that HDL are able to counteract the inhibitory effect of oxidised LDL (ox-LDL) on endothelium-dependent vasorelaxation [5].

Endothelium dysfunction, a predictor of cardiovascular events [6], has been observed in patients with type 2 diabetes mellitus [7,8]. Due to qualitative modifications, 
HDL from type 2 diabetic patients may be less protective against impairments of endothelium-dependent vasorelaxation than HDL from healthy subjects. To test this hypothesis, we investigated whether HDL from type 2 diabetic patients show the same ability as HDL from normolipidaemic and normoglycaemic control subjects to protect endothelium-dependent vasorelaxation from the inhibitory effect of ox-LDL. In order to study the effect of HDL separately, our work was performed on isolated rings of rabbit aorta, a previously validated model $[5,9]$. In addition we measured the activity of paraoxonase (PON), an HDL-associated enzyme with an anti-oxidant role due to its ability to hydrolyse some oxidised lipids.

\section{Subjects and methods}

\section{Subjects}

We included 16 type 2 diabetic patients and 13 normolipidaemic and normoglycaemic control subjects in this protocol, which was approved by the Ethics Committee of Dijon University Hospital, with written informed consent obtained from each subject before the study. Type 2 diabetic and control subjects were not taking any antioxidants or any drugs that could affect lipid metabolism except hypoglycaemic drugs for type 2 diabetic patients. Of the diabetic patients, three patients were being treated by sulfonylureas, four by glinides, two by metformin, three by sulfonylureas and metformin, two by sulfonylurea and acarbose, and two by sulfonylureas, metformin and acarbose. No type 2 diabetic subject was insulin-treated or taking thiazolidinediones. Diabetes duration was $9.13 \pm$ 6.85 years. Three diabetic patients had microalbuminuria $>30 \mathrm{mg} / \mathrm{l}$, one had retinopathy and two had neuropathy. Nine diabetic patients had high blood pressure and five had cardiovascular disease or macroangiopathy. Nine diabetic patients were taking one or several of the following drugs: angiotensin converting enzyme inhibitors, beta blocking drugs, calcium channel blockers, antithrombotic drugs. Metabolic syndrome was excluded in control subjects according to the criteria of the National Cholesterol Education Program Adult Treatment Panel III report (NCEP-ATP III) [10].

\section{Chemical compounds}

Acetylcholine, arterenol hydrochloride, sodium nitro-prusside, butylated hydroxytoluene and EDTA were purchased from Sigma Chemical (Saint Quentin Fallavier, France).
Preparation of lipoproteins

The different lipoprotein subclasses were isolated from serum or plasma by sequential flotation ultracentrifugation, according to their density $(d)$. The LDL $(1.019<d<1.063$ $\mathrm{g} / \mathrm{ml}$ ) used to form ox-LDL were prepared in a 70.Ti rotor on an ultracentrifuge (XL-90; Beckman, Palo Alto, CA, USA) by sequential flotation ultracentrifugation from $170 \mathrm{ml}$ of fresh citrated plasma drawn from normolipidaemic donors. Densities of plasma were adjusted by adding potassium bromide. After a 15-hour run at $184,000 \mathrm{~g}$, the top fraction of the sample containing lipoproteins with $d<1.019 \mathrm{~g} / \mathrm{ml}$ was removed. Finally, LDL were prepared from the infranatant adjusted to a $1.063 \mathrm{~g} / \mathrm{ml}$ density by a 24 -hour run at $149,000 \mathrm{~g}$. HDL (1.063< $d<1.21 \mathrm{~g} / \mathrm{ml}$ ) of control and type 2 diabetic subjects were prepared from $20 \mathrm{ml}$ of EDTA plasma in a 70.Ti rotor on an ultracentrifuge (Beckman). After a 20-hour run at $184,000 \mathrm{~g}$, the top fraction of the sample containing lipoproteins with $d<1.063 \mathrm{~g} / \mathrm{ml}$, was removed. Finally, HDL were prepared from the infranatant adjusted to a $1.21 \mathrm{~g} / \mathrm{ml}$ density by a 24-hour run at $184,000 \mathrm{~g}$. The different lipoprotein fractions were dialysed overnight against a $10 \mathrm{mmol} / 1$ Tris, $150 \mathrm{mmol} / \mathrm{l} \mathrm{NaCl}, \mathrm{pH} 7.4$ buffer (TBS). To avoid oxidation, native lipoproteins were supplemented with EDTA (final concentration, $200 \mu \mathrm{mol} / \mathrm{l}$ ) and butylated hydroxytoluene (final concentration, $20 \mu \mathrm{mol} / \mathrm{l})$ and kept at $4^{\circ} \mathrm{C}$.

\section{Oxidative modification of LDL}

The oxidation of LDL was performed by incubating freshly prepared LDL, adjusted to a $1.2 \mathrm{~g} / 1$ final concentration of proteins in TBS, with a copper sulfate solution (final concentration, $5 \mu \mathrm{mol} / \mathrm{l})$ for $24 \mathrm{~h}$ at $37^{\circ} \mathrm{C}$. At the end, oxidation was stopped by adding EDTA (final concentration, $200 \mu \mathrm{mol} / \mathrm{l}$ ) and butylated hydroxytoluene (final concentration, $20 \mu \mathrm{mol} / \mathrm{l}$ ) and ox-LDL were kept at $4^{\circ} \mathrm{C}$ [9].

\section{Serum and HDL biochemical measurements}

Glycaemia was measured on a Vitros analyser (Ortho Clinical Diagnostics, Rochester, NY, US). Total and HDL cholesterol, TG, proteins, apolipoprotein (apo)AI and apoB were quantitated on a Dimension analyser with dedicated reagents (Dade Behring, Newark, DE, USA). LDL-cholesterol was calculated using the Friedewald formula. Fructosamine, free cholesterol and phospholipids were measured on the same analyser with Wako reagents (Wako, Neuss, Germany) for free cholesterol and phospholipids, and $\mathrm{ABX}$ Diagnostics reagents (Montpellier, France) for fructosamine. $\mathrm{HbA}_{1 \mathrm{c}}$ was performed by high performance liquid chromatography on a Variant II (BioRad, Richmond, CA, US) (normal range: 4.0-6.0\%). 
Measurement of paraoxonase activity

PON activity was measured as already described in fresh HDL samples within 2 days of isolation [11]. It was assayed in tubes containing Tris- $\mathrm{HCl}$ buffer $(150 \mathrm{mmol} / \mathrm{l}$ $\mathrm{NaCl}, 1 \mathrm{mmol} / \mathrm{l} \mathrm{CaCl}$, $\mathrm{pH} 7.4)$ and $100 \mu \mathrm{g}$ proteins of HDL (final volume $800 \mu \mathrm{l}$ ). The reaction was initiated by adding paraoxon $(1 \mathrm{mmol} / \mathrm{l})$ and the absorbance monitored at $412 \mathrm{~nm}$ for $1 \mathrm{~h}$. The amount of 4-nitrophenol formed was calculated from the molar extinction coefficient $\left(128,00 \mathrm{~mol}^{-1} \mathrm{~cm}^{-1}\right)$. PON activity was expressed as nmol of 4-nitrophenol formed per minute and $\mathrm{mg}$ of apoAI, under the experimental conditions.

Vasoreactivity on rabbit aorta rings

Vasoreactivity experiments were performed on rabbit (Charles River, L'Arbresle, France) aorta rings as previously described [9]. Our protocol has been approved by the local Ethics Committee for animals at Dijon University. The principles of laboratory animal care were followed. Aortic rings were first contracted by $0.3 \mu \mathrm{mol} / 1$ arterenol hydrochloride, a concentration giving $75 \%$ of the maximal contraction. Then the rings were relaxed by cumulative additions of acetylcholine in the $1 \mathrm{nmol} / 1$ to $0.01 \mathrm{mmol} / \mathrm{l}$ concentration range. After wash-out and a 30-min recovery period, aortic rings were incubated for $2 \mathrm{~h}$ either with oxLDL or HDL or both. The concentration of proteins in the organ bath (native or oxidised lipoproteins) was constantly $1 \mathrm{~g} / \mathrm{l}$ for each lipoprotein subclass, as used in many previous studies conducted with rabbit aortic rings $[5,9$, 12]. At the end of the incubation period, aortic segments were again contracted with arterenol hydrochloride and progressively relaxed with acetylcholine or with sodium nitroprussiate. Sodium nitroprussiate, a nitric oxide (NO) donor, was used to check that the observed defects in vasodilatation were endothelium-dependent.

The maximal relaxation (Emax) induced by acetylcholine and expressed as a percentage of the contraction to arterenol hydrochloride $(0.3 \mu \mathrm{mol} / 1)$ was determined from experimental data. To limit variations of results due to intrinsic properties of vessels, experiments were performed in quadruplicate on aorta rings from four different rabbits, and results were averaged.

\section{Statistical analysis}

Statistical analysis was performed using Statview statistic software. Data are expressed as mean \pm SD. The comparison of data from type 2 diabetic subjects and controls was performed by the Mann-Whitney $U$ test. The maximal relaxation values obtained in two different conditions and the same experiment were compared by the nonparametric Wilcoxon matched-pair test. Correlation coefficients were performed by simple linear regression analysis. We also performed a multiple regression analysis in which the dependent variable was the maximal vasorelaxation and the independent variables were HDL TG percentage, HDL fructosamine:protein ratio, plasma $\mathrm{TG}$, age, sex, diabetes duration and PON. A $p$ value $<0.05$ was considered to be statistically significant.

\section{Results}

Clinical and biochemical characteristics

The clinical and biochemical characteristics of type 2 diabetic and control subjects are shown in Table 1. Each of our control subjects had a fasting glycaemia $<6.10 \mathrm{mmol} / 1$. We recruited 16 type 2 diabetic patients with a broad range of glycaemic control, i.e. with fasting glycaemia at 4.66 to $18.57 \mathrm{mmol} / 1$ and $\mathrm{HbA}_{1 \mathrm{c}}$ of 6.0 to $13.3 \%$. Total plasma and LDL cholesterol did not differ between the two groups. Compared to the control group, plasma TG level was 1.8fold higher in the type 2 diabetic group $(p<0.05)$, whereas serum HDL cholesterol and apoAI concentrations were $26 \%(p<0.01)$ and 14\% $(p<0.05)$ lower, respectively.

\section{HDL composition}

Table 2 illustrates the composition of HDL in the two groups of subjects. Compared to control subjects, type 2 diabetic patients exhibited an 1.7-fold higher TG proportion in HDL $(p<0.01)$, whereas other lipid components, total proteins and apoAI were not significantly different. The HDL TG:apoAI ratio was 1.5 -fold higher $(p<0.05)$ in type 2 diabetic patients than in control subjects and the HDL fructosamine:total protein ratio was 2.7-fold higher $(p<0.005)$. PON activity was not statistically different between the two groups.

Table 1 Clinical and biochemical characteristics of type 2 diabetic and control subjects

\begin{tabular}{lll}
\hline & $\begin{array}{l}\text { Control subjects } \\
(n=13)\end{array}$ & $\begin{array}{l}\text { Type 2 diabetic } \\
\text { patients }(n=16)\end{array}$ \\
\hline Age (years) & $57 \pm 13$ & $63 \pm 13$ \\
Sex (male/female) & $7 / 6$ & $8 / 8$ \\
BMI $\left(\mathrm{kg} / \mathrm{m}^{2}\right)$ & $23.6 \pm 1.4$ & $30.5 \pm 5.4^{\mathrm{c}}$ \\
Waist circumference $(\mathrm{cm})$ & $83.4 \pm 9.1$ & $108.5 \pm 13.3^{\mathrm{c}}$ \\
Fasting glycaemia $(\mathrm{mmol} / \mathrm{l})$ & $5.48 \pm 0.55$ & $9.19 \pm 4.20^{\mathrm{b}}$ \\
HbA 1 c $(\%)$ & $\mathrm{ND}$ & $9.3 \pm 3.0$ \\
Total cholesterol $(\mathrm{mmol} / \mathrm{l})$ & $5.68 \pm 0.78$ & $5.49 \pm 1.49$ \\
HDL-cholesterol $(\mathrm{mmol} / \mathrm{l})$ & $1.50 \pm 0.35$ & $1.11 \pm 0.30^{\mathrm{a}}$ \\
LDL-cholesterol $(\mathrm{mmol} / \mathrm{l})$ & $3.62 \pm 0.70$ & $3.42 \pm 1.12$ \\
TG (mmol/l) & $1.18 \pm 0.57$ & $2.08 \pm 1.50^{\mathrm{b}}$ \\
Apo AI $(\mathrm{g} / \mathrm{l})$ & $1.46 \pm 0.23$ & $1.25 \pm 0.29^{\mathrm{b}}$ \\
Apo B $(\mathrm{g} / \mathrm{l})$ & $1.03 \pm 0.28$ & $1.09 \pm 0.32$ \\
\hline
\end{tabular}

Values are expressed as mean $\pm \mathrm{SD}$

$N D$, Not determined

${ }^{\mathrm{a}} p<0.05 ;{ }^{\mathrm{b}} p<0.005 ;{ }^{\mathrm{c}} p<0.001$ 
Table 2 HDL composition in control and type 2 diabetic subjects

\begin{tabular}{lcc}
\hline & $\begin{array}{c}\text { Control subjects } \\
(n=13)\end{array}$ & $\begin{array}{c}\text { Type 2 diabetic } \\
\text { patients }(n=16)\end{array}$ \\
\hline Protein (\%) & $49.6 \pm 6.2$ & $51.5 \pm 4.7$ \\
Phospholipid (\%) & $21.4 \pm 3.7$ & $18.5 \pm 3.5$ \\
Free cholesterol (\%) & $2.8 \pm 0.5$ & $2.7 \pm 0.4$ \\
Cholesteryl ester (\%) & $23.2 \pm 2.6$ & $22.9 \pm 4.6$ \\
TG (\%) & $3.1 \pm 1.4$ & $5.3 \pm 2.2^{\mathrm{b}}$ \\
ApoAI (\%) & $38.5 \pm 5.3$ & $42.5 \pm 10.3$ \\
TG/apoAI (mmol/g) & $0.084 \pm 0.040$ & $0.124 \pm 0.052^{\mathrm{a}}$ \\
Fructosamine/total & $2.39 \pm 0.42$ & $6.48 \pm 3.25^{\mathrm{c}}$ \\
proteins ( $\mu \mathrm{mol} / \mathrm{mg})$ & & \\
PON activity & $1.39 \pm 0.89$ & $1.31 \pm 0.80$ \\
$\quad\left(\mathrm{nmol} \mathrm{min}{ }^{-1} \mathrm{mg}^{-1}\right.$ apoAI) & & \\
\hline
\end{tabular}

Values are expressed as mean $\pm \mathrm{SD}$

HDL composition is expressed as the percentage of total HDL mass ${ }^{\mathrm{a}} p<0.05 ;{ }^{\mathrm{b}} p<0.01 ;{ }^{\mathrm{c}} p<0.005$

Oxidised LDL influence on endothelium-dependent vasorelaxation

In accordance with previous data, pre-incubation of isolated arterial segments with ox-LDL inhibited the endothelium-dependent relaxation induced by acetycholine, the maximal relaxation to acetycholine decreasing from $99.3 \pm 5.2 \%$ in segments incubated with Krebs solution to $58.2 \pm 14.6 \%$ in segments treated with ox-LDL $(p<0.0001)$ (Fig. 1).

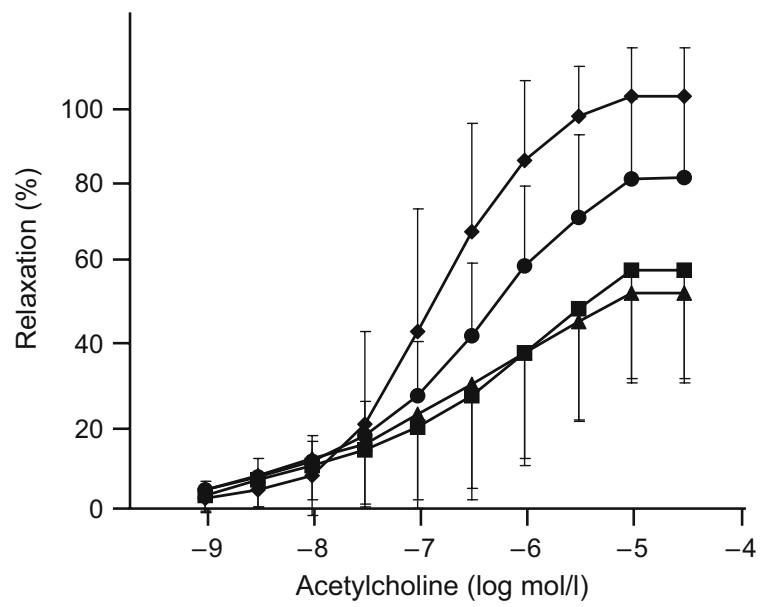

Fig. 1 Influence of HDL from type 2 diabetic and control subjects on the inhibitory effect of ox-LDL on endothelium-dependent vasorelaxation in rabbit aorta rings. The graph shows mean concentration-response curves for acetylcholine in aortas contracted with $0.3 \mu \mathrm{mol} / 1$ arterenol hydrochloride and pre-incubated for $2 \mathrm{~h}$ either with ox-LDL alone (1 g protein/l) (squares), or with ox-LDL and HDL from control subjects (circles), or with ox-LDL and HDL from type 2 diabetic patients $(1 \mathrm{~g}$ protein/l) (triangles) or in Krebs buffer (diamonds). Values represent means $\pm \mathrm{SD}$
Ability of HDL to counteract the inhibitory effect of oxidised LDL on endothelium-dependent vasodilation

Compared to the maximal relaxation obtained in segments pre-incubated in Krebs buffer, no significant difference was observed between segments pre-incubated with HDL from control subjects or from type 2 diabetic subjects $(\mathrm{Emax}=92.8 \pm 4.0$ vs $99.3 \pm 5.2$ and $92.4 \pm 8.4$ vs $99.3 \pm 5.2 \%$, respectively) (data not shown). The maximal relaxation of rabbit aortic rings was significantly greater after preincubation with HDL from control subjects + ox-LDL than after preincubation with ox-LDL alone $(\operatorname{Emax}=77.6 \pm 12.9$ vs $59.5 \pm 7.7 \%, p<0.01)$ (Fig. 1). In contrast, the addition of HDL from type 2 diabetic patients to ox-LDL did not modify the maximal relaxation, compared to incubation with ox-LDL $($ Emax $=52.4 \pm 20.4$ vs $57.2 \pm 18.7 \%$, NS) (Fig. 1).

\section{Linear regression analysis}

In type 2 diabetic patients the Emax obtained after coincubation of the vessels with ox-LDL and HDL (Emax [ox-LDL+HDL]) was strongly and negatively correlated with the percentage of TG in HDL and the HDL TG:apo AI ratio ( $r=-0.71, p<0.005$ and $r=-0.60, p<0.05$, respectively) (Fig. 2a,b). In control subjects, such a correlation was not observed $(r=0.138, \mathrm{NS})$. Emax (ox-LDL+HDL) was not
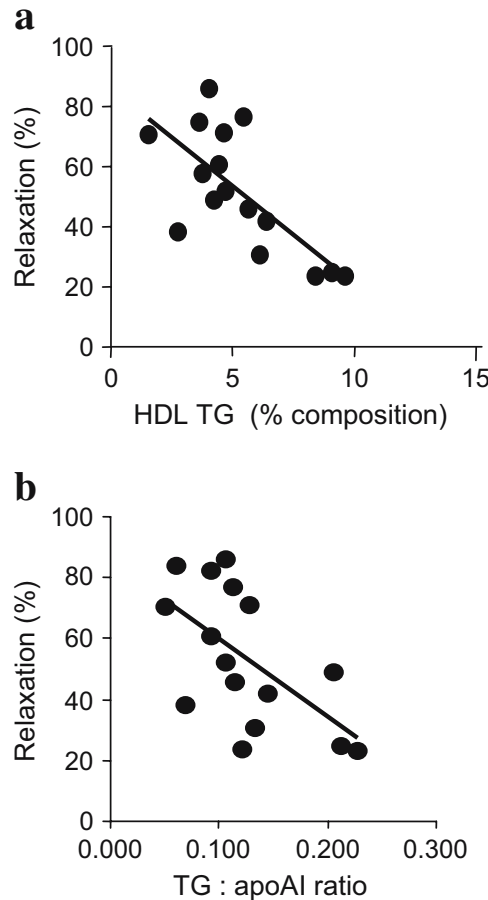

Fig. 2 Correlation between a HDL-TG and b TG:apoAI ratio, and the maximal endothelium-dependent relaxation on rabbit aortic rings after preincubation with HDL from type 2 diabetic subjects and oxidised LDL. $r=-0.71, p=0.0019$ (a); $r=-0.60, p=0.0147$ (b) 
correlated with the percentage in HDL-free or esterified cholesterol, phospholipids or proteins, either in type 2 diabetic patients or in control subjects. No significant correlation was observed between the HDL fructosamine: total protein ratio or PON activity and Emax (ox-LDL+ HDL) in type 2 diabetic patients or controls. In multiple regression analysis, including HDL TG percentage, the HDL fructosamine:protein ratio, plasma TG, age, sex, diabetes duration, and PON, the only parameter that was significantly correlated to Emax was HDL TG percentage $(r=-0.91, p=0.0134)$.

\section{Discussion}

In the present study, we demonstrated for the first time that the ability of HDL to protect the vessel from ox-LDLinduced inhibition of endothelium-dependent vasorelaxation is impaired in type 2 diabetic patients. Moreover, the ability of HDL to counteract the inhibitory effect of oxLDL on vasorelaxation is inversely correlated with HDL TG content. In contrast, no significant correlation was observed between the HDL fructosamine:total protein ratio or PON activity and Emax (ox-LDL+HDL) in type 2 diabetic patients or control subjects.

The inhibitory effect of ox-LDL and some of their specific compounds such as derivatives of cholesterol oxidised in position 7 or lysophophatidylcholine on endothelium-dependent vasorelaxation is mainly related to a decreased bioavailability of NO [13-17]. HDL are likely to counteract the inhibitory effect of ox-LDL on endotheliumdependent vasorelaxation both by a direct and an indirect way. HDL inhibit the LDL oxidation process by removing lipid peroxides and oxidised derivatives of cholesterol from LDL via cholesteryl ester transfer protein [18]. The two HDL-associated enzymes, PON and platelet-activated factor-acetyl hydrolase (PAF-AH) hydrolyse oxidised cholesterol or hydroperoxides [19-21]. Furthermore, HDL prevent the depletion in free cholesterol of endothelial cell caveolae induced by ox-LDL, thus allowing eNOS to stay in caveolae, where it can be activated by vasodilatory ligands of $\mathrm{G}$ protein-coupled receptors [22]. On the other hand, HDL can stimulate NO production. eNOS activity can be upregulated by HDL through regulation of phosphorylation sites [23]. Different signalling pathways are likely to be involved in this process. First, the binding of HDL to scavenger receptor BI has been demonstrated to activate Akt and mitogen-activated protein kinases, which in turn phosphorylate eNOS [24]. Secondly, the binding of HDL to the lysophospholipid receptor $\mathrm{S}_{3} \mathrm{P}_{3}$ activates $\mathrm{Ca}^{2+}$-calmodulin-dependent kinases and Akt with consecutive activation of eNOS [25]. Finally, HDL can increase the expression of the gene encoding eNOS [26].

The inability of HDL from type 2 diabetic patients to counteract the inhibitory effect of ox-LDL on endothelium- dependent vasodilatation indicates that one or several of the HDL protective effects detailed above are deficient.

In previously published works, HDL from patients with metabolic syndrome or type 2 diabetes mellitus were less protective against LDL oxidation than HDL from healthy subjects, and the antioxidative capacity of HDL was inversely correlated with plasma and HDL TG $[11,27,28]$. The lesser antioxidant capacity of HDL from type 2 diabetic patients is likely to contribute to the lesser ability of these particles to counteract the inhibitory effect of ox-LDL on endothelium-dependent vasorelaxation. The mechanisms underlying the decreased antioxidant capacity of HDL from type 2 diabetic patients are not well understood. However, TGs appear to play an important role, since a strong inverse correlation was found between the content of HDL in TGs and their antioxidant properties in several studies [11, 27, 28]. Moreover, it has recently been demonstrated that the replacement of cholesteryl esters by TGs in HDL abrogates the HDL-mediated protection against LDL oxidation [28].

PON and PAF-AH activities have inconstantly been found to be decreased and related to HDL antioxidant capacity in type 2 diabetic patients [11, 27-29]. Interestingly, in type 2 diabetic patients, the antioxidant capacity of HDL can be decreased, whereas PON and PAF-AH activities are similar to those measured in controls [11], indicating that in type 2 diabetes mellitus other factors than the decrease of PON and PAF-AH activities are likely to impair HDL antioxidant activity. Thus, in our study, a normal PON activity in HDL from type 2 diabetic patients does not preclude impairment of the antioxidant capacity of these HDL.

During the few last years it was found that HDL could also be oxidised [30]. It is noteworthy that we observed no impairment of Emax with HDL from type 2 diabetic patients alone and that Emax was quite similar to that measured with HDL from control subjects, indicating that HDL from our type 2 diabetic patients were not able to decrease NO bioavailability by themselves, and that initially they were probably not highly oxidised.

The inability of HDL from type 2 diabetic patients to counteract the inhibitory effect of ox-LDL on endotheliumdependent vasorelaxation could also be related to a lesser capacity to induce NO synthesis. Experiments on reconstituted HDL particles demonstrated that changes to the HDL content in TG modulate the conformation of apoAI and the expression of epitopes all along the molecule $[31,32]$. Therefore, the TG enrichment of HDL particles usually observed in type 2 diabetic patients could modify apoAI conformation and decrease the binding of HDL particles to the cell surface receptors implicated in the signalling pathways that activate eNOS. This hypothesis needs to be confirmed by further experimentation.

The fact that we recruited patients with a wide range of glycaemic control allowed us to search for a possible relationships between HDL glycation and HDL vasorelaxant effect. We did not observe any correlation between 
these two parameters, which suggests that HDL glycation does not play an important role in the lesser vasorelaxant effect of HDL from type 2 diabetic patients, compared to the TG enrichment.

A limitation of our study is that we did not measure PAF$\mathrm{AH}$ and lecithin cholesterol acyltransferase activities. However, in previous studies performed in type 2 diabetic patients or insulin-resistant subjects no correlation could be found between the activity of these two enzymes and the decreased antioxidant properties of HDL $[10,26,27]$. Therefore, it is unlikely that we could have explained the decreased vasorelaxant effect of HDL from type 2 diabetic patients by a decrease in PAF-AH and lecithin cholesterol acyltransferase activities.

In conclusion, the present study demonstrates that the ability of HDL from type 2 diabetic patients to counteract the inhibitory effects of ox-LDL on endothelium-dependent vasorelaxation is impaired, and is highly inversely correlated with the TG content of HDL. As endothelial dysfunction is likely to favour the early development of atherosclerotic lesions, these findings provide further evidence that HDL in patients with type 2 diabetes mellitus has a lower atheroprotective effect than that in control subjects; they also provide further evidence for the deleterious effect of the hypertriglyceridaemia frequently associated with this disease.

Acknowledgements The authors wish to thank D. Battault, E. Niot and L. Princep for their expert technical assistance. This study was supported by the Institut National de la Santé et de la Recherche Médicale (INSERM), the Université de Bourgogne and the Conseil Régional de Bourgogne.

\section{References}

1. Biesbroeck RC, Albers JJ, Wahl PW, Weinberg CR, Bassett ML, Bierman EL (1982) Abnormal composition of high density lipoproteins in non-insulin-dependent diabetics. Diabetes 31:126-131

2. Taskinen MR (1992) Quantitative and qualitative lipoprotein abnormalities in diabetes mellitus. Diabetes 41(Suppl 2):12-17

3. Bisoendial RJ, Hoving GK, Levels JHM et al (2003) Restoration of endothelial function by increasing high-density lipoprotein in subjects with isolated low high-density lipoprotein. Circulation 107:2944-2948

4. Spieker LE, Sudano I, Hurlimann D et al (2002) High-density lipoprotein restores endothelial function in hypercholesterolemic men. Circulation 105:1399-1402

5. Matsuda Y, Hirata K, Inoue N et al (1993) High density lipoprotein reverses inhibitory effect of oxidized low density lipoprotein on endothelium-dependent arterial relaxation. Circ Res 72:1103-1109

6. Halcox JP, Schenke WH, Zalos G et al (2002) Prognostic value of coronary vascular endothelial dysfunction. Circulation 106:653-658

7. Schachinger V, Britten MB, Zeiher AM (2000) Prognostic impact of coronary vasodilator dysfunction on adverse longterm outcome of coronary heart disease. Circulation 101: 1899-1906
8. McVeigh GE, Brennan GM, Johnston GD et al (1992) Impaired endothelium-dependent and independent vasodilation in patients with type 2 (non-insulin-dependent) diabetes mellitus. Diabetologia 35:771-776

9. Deckert V, Perségol L, Viens L et al (1997) Inhibitors of arterial relaxation among components of human oxidized low-density lipoproteins. Cholesterol derivatives oxidized in position 7 are potent inhibitors of endothelium-dependent relaxation. Circulation 95:723-731

10. Expert panel on detection, evaluation and treatment of high blood cholesterol in adults (Adult Treatment Panel III) (2001) Executive summary of The Third Report of The National Cholesterol Education Program (NCEP). JAMA 285:2486-2497

11. Gowri MS, Van der Westhuysen DR, Bridges SR, Anderson JW (1999) Decreased protection by HDL from poorly controlled type 2 diabetic subjects against LDL oxidation may be due to the abnormal composition of HDL. Arterioscler Thromb Vasc Biol 19:2226-2233

12. Jacobs M, Plane F, Bruckdorfer RK (1990) Native and oxidized low-density lipoproteins have different inhibitory effects on endothelium-derived relaxing factor in the rabbit aorta. $\mathrm{Br}$ J Pharmacol 100:21-26

13. Deckert V, Brunet A, Lantoine F et al (1998) Inhibition by cholesterol oxides of $\mathrm{NO}$ release from human vascular endothelial cells. Arterioscler Thromb Vasc Biol 18:1054-1060

14. Kugiyama K, Kerns SA, Morrisett JD, Roberts R, Henry PD (1990) Impairment of endothelium-dependent arterial relaxation by lysolecithin in modified low-density lipoproteins. Nature 344:160-162

15. Jessup W (1996) Oxidized lipoproteins and nitric oxide. Curr Opin Lipidol 7:274-280

16. Wever R, Stroes E, Rabelink TJ (1998) Nitric oxide and hypercholesterolemia: a matter of oxidation and reduction? Atherosclerosis 137:51-60

17. Blair A, Shaul PW, Yuhanna IS, Conrad PA, Smart EJ (1999) Oxidized low density lipoprotein displaces endothelial nitricoxide synthase (eNOS) from plasmalemmal caveolae and impairs eNOS activation. J Biol Chem 274:32512-32519

18. Christison JK, Rye K-A, Stocker R (1995) Exchange of oxidized cholesteryl linoleate between LDL and HDL by cholesteryl ester transfer protein. J Lipid Res 36:2017-2026

19. Mackness MI, Arrol S, Abbott C, Durrington P (1993) Protection of low-density lipoprotein against oxidative modification by high-density lipoprotein associated paraoxonase. Atherosclerosis 104:129-135

20. Watson AD, Berliner JA, Hama SY et al (1995) Protective effect of high density lipoprotein associated paraoxonase. J Clin Invest 96:2882-2891

21. Watson AD, Navab M, Hama SY et al (1995) Effect of plateletactivating factor-acetylhydrolase on the formation and action of minimally oxidized low density lipoprotein. J Clin Invest 95: $774-782$

22. Uittenbogaard A, Shaul PW, Yuhanna IS, Blair A, Smart EJ (2000) High density lipoprotein prevents oxidized low density lipoprotein-induced inhibition of endothelial nitric-oxide synthase localization and activation in caveolae. J Biol Chem 275: $11278-11283$

23. Drew BG, Fidge NH, Gallon-Beaumier G, Kemp BE, Kingwell BA (2004) High-density lipoprotein and apolipoprotein AI increase endothelial NO synthase activity by protein association and multisite phosphorylation. Proc Natl Acad Sci USA 101:6999-7004

24. Mineo C, Yuhanna IS, Quon MJ, Shaul PW (2003) High density lipoprotein-induced endothelial nitric-oxide synthase activation is mediated by Akt and MAP kinases. J Biol Chem 278:9142-9149 
25. Nofer JR, van der Giet M, Tölle M et al (2004) HDL induces NO-dependent relaxation via lysophospholipid receptor S1P3. $\mathrm{J}$ Clin Invest 113:569-581

26. Kuvin JT, Rämet ME, Patel AR, Pandian NG, Mendelson ME, Karas RH (2002) A novel mechanism for the beneficial vascular effects of high-density lipoprotein cholesterol: enhanced vasorelaxation and increased endothelial nitric oxide synthase expression. Am Heart J 144:165-172

27. Hansel B, Giral P, Nobecourt E et al (2004) Metabolic syndrome is associated with elevated oxidative stress and dysfunctional dense high-density lipoprotein particles displaying impaired antioxidative activity. J Clin Endocrinol Metab $89: 4963-4971$

28. Nobecourt E, Jacqueminet S, Hansel B et al (2005) Defective antioxidative activity of small dense HDL3 particles in type 2 diabetes: relationship to elevated oxidative stress and hyperglycaemia. Diabetologia 48:529-538
29. Kopprasch S, Pietzsch J, Kuhlisch E, Graessler J (2003) Lack of association between serum paraoxonase 1 activities and increased oxidized low-density lipoprotein levels in impaired glucose tolerance and newly diagnosed diabetes mellitus. J Clin Endocrinol Metab 88:1711-1716

30. Thomas MJ, Chen Q, Zabalawi M et al (2001) Is the oxidation of high-density lipoprotein lipids different than the oxidation of low-density lipoprotein lipids? Biochemistry 13:1719-1724

31. Curtiss LK, Bonnet DJ, Rye KA (2000) The conformation of apolipoprotein A-I in high-density lipoproteins is influenced by core lipid composition and particle size: a surface plasmon resonance study. Biochemistry 39:5712-5721

32. Braschi S, Coffill CR, Neville TA, Hutt DM, Sparks DL (2001) Effect of acylglyceride content on the structure and function of reconstituted high density lipoprotein particles. J Lipid Res 42:79-87 